\title{
A wave and finite element methodology for structural identification in layered composite structures
}

\author{
Rilwan Kayode Apalowo ${ }^{1, *}$, Dimitrios Chronopoulos ${ }^{1}$, and Muhammed Malik ${ }^{1}$ \\ ${ }^{1}$ Institute for Aerospace Technology \& The Composites Group, The University of Nottingham, NG7 \\ 2RD, UK
}

\begin{abstract}
In this work we present for the first time an approach for identifying the geometric and material characteristics of layered composite structures through an inverse wave and finite element approach. More specifically, this Non-Destructive Evaluation (NDE) approach is able to recover the thickness, density, as well as all independent mechanical characteristics such as the tensile and shear moduli for each layer of the composite structure under investigation. This is achieved through multi-frequency single shot measurements. It is emphasized that the success of the approach is independent of the employed excitation frequency regime, meaning that both structural dynamics and ultrasound frequency spectra can be employed. It is demonstrated that more efficient convergence of the identification process is attained closer to the bending-to-shear transition range of the layered structure. Since a full FE description is employed for the periodic composite, the presented approach is able to account for structures of arbitrary complexity. The procedure is applied to a sandwich panel with composite facesheets and results are compared with two wave-based characterization techniques: the Inhomogeneous Wave Correlation method and the Transition Frequency Characterization method. Numerical simulations and experimental results are presented to verify the robustness of the proposed method.
\end{abstract}

\section{Introduction}

Composites are widely used in modern industry, due to their low density and high dynamic and static performances. However, the verification and Non-Destructive Evaluation (NDE) of the actual mechanical properties of the assembled layered structure remains a very much open engineering challenge. In a broad context, 'system identification' refers to the extraction of information about the system behavior directly from experimental data. Over the past decades, different system identification methods in the time domain [1], frequency domain [2] and time-frequency domain [3] have been proposed.

Many methods have been developed to perform material characterization in composites. One can cite the experimental method for the characterization of Nomex cores [4], or the vibratory identification technique proposed in Matter et al. [5]. Finite Element (FE) based wave methods assume a full 3D displacement field and are therefore capable of capturing the entirety of wave motion types in the waveguide under investigation in a very accurate and

\footnotetext{
*e-mail: Rilwan.Apalowo@nottingham.ac.uk
} 
efficient manner. FE-based wave propagation within periodic structures was firstly considered in [6]. The Wave and Finite Element (WFE) method was introduced in [7, 8] in order to facilitate the post-processing of the eigenproblem solutions. The WFE has recently found applications in predicting the vibroacoustic and dynamic performance of composite panels and shells [9-11], with complex periodic structures $[12,13]$ having been investigated. The variability of vibroacoustic transmission through layered structures [14-16], as well as structural identification [17] have also been considered.

The principal novel contribution of this work is the development of a comprehensive methodology coupling periodic structure theory to FE in order to identify the characteristics of each individual layer of a composite structure through experimental measurements on the entire structure. The paper is organised as follows: In Sec. 2 the FE computational scheme for predicting wave propagation in multilayered structures is presented. In Sec. 3, experimental and numerical case studies are presented for validating the exhibited identification approach. Conclusions are eventually drawn in Sec. 4.

\section{An inverse wave and finite element methodology for structural identification}

An arbitrarily complex and periodic in the $x$ direction waveguide is illustrated in Fig.1. The structure may comprise an arbitrary number of layers which may be anisotropic. The identifiable properties include the thickness, density as well as the material characteristics of each individual layers.

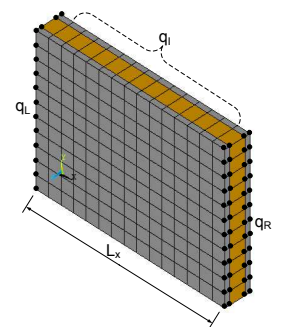

Figure 1. Caption of the WFE modelled composite waveguide with the left and right side nodes $\mathbf{q}_{L}, \mathbf{q}_{R}$ bullet marked. The range of interior nodes $\mathbf{q}_{I}$ is also illustrated.

\subsection{Obtaining the reference wave characteristics}

The excitation signals are quasi-monochromatic burst of amplitude $U_{0}$, centred around frequency $f_{0}$ and involving a number of $n_{0}$ cycles. Input signals are windowed so that the input signal is defined by $u(t)=U_{0} \sin \left(\frac{\pi f_{0} t}{n_{0}}\right) \sin \left(2 \pi f_{0} t\right)$ for $0 \leq t \leq \frac{n_{0}}{f_{0}}$ and $u(t)=0$ for $t>\frac{n_{0}}{f_{0}}$.

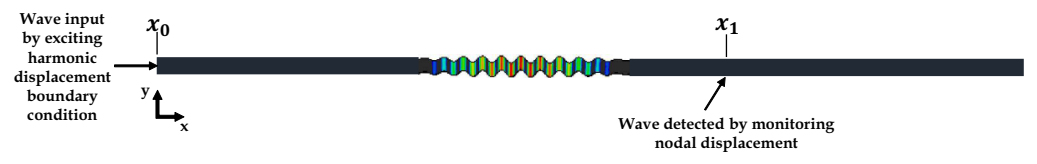

Figure 2. Configuration for obtaining the reference wave characteristics to be compared with the WFE ones 
An illustration of the configuration is depicted in Fig.2. The waveguide is excited at a specified central harmonic signal of frequency $f_{0}$ at a location $x=x_{0}$ and the signal is monitored at location $x=x_{1}$, after which the signal has travelled over a distance of $L=x_{1}-$ $x_{0}$. Once the experimental or numerical signal measurements are logged, the wavenumbers and group velocities of the excited waves can be easily determined.

Time histories are initially registered at the excitation and monitoring locations. The maximum amplitudes of the time history signals $\mathbf{x}(t)$ are obtained from the Hilbert Transforms $H[\mathbf{x}(t)]$ of the acquired signals in the time domain. The total Time of Flight of the wave signal from the point of excitation to the monitoring point is measured as the time difference $t\left(x_{1}\right)-t\left(x_{0}\right)$ between the maximum amplitudes of the excited and the monitored signal envelopes. In ultrasonic NDE, the wavenumber of the wave package is straightforward to obtain as:

$$
k=\frac{\omega}{c_{p}}
$$

where the phase velocity of the signal $c_{p}$ can be obtained from its ToF and its propagation distance $L$. It is noted that the phase velocity for a non-dispersive wave is equal to its group velocity.

\subsection{Structural identification methodology}

\subsubsection{Considerations on the forward wave FE model}

Linear elastic wave propagation is considered in the $x$ direction of the arbitrarily layered structural waveguide of Fig.1. Following the analysis presented in [17], the free wave propagation eigenproblem can be expressed as

$$
\gamma\left\{\begin{array}{l}
\mathbf{q}_{\mathbf{L}}^{(s)} \\
\mathbf{f}_{\mathbf{L}}^{(s)}
\end{array}\right\}=\mathbf{T}\left\{\begin{array}{l}
\mathbf{q}_{\mathbf{L}}^{(s)} \\
\mathbf{f}_{\mathbf{L}}^{(s)}
\end{array}\right\}
$$

whose eigenvalues $\gamma_{\omega}$ and eigenvectors $\boldsymbol{\phi}_{\omega}=\left\{\begin{array}{l}\boldsymbol{\phi}_{q} \\ \boldsymbol{\phi}_{f}\end{array}\right\}_{\omega}$ solution sets provide a comprehensive description of the propagation constants at a specified angular frequency $\omega$.

\subsubsection{Formulation of the identification objective function}

Since each resulting $j_{\text {th }}$ eigenvalue (propagation constants for each wave type) can be expressed as $\gamma_{j, f e}=\mathrm{e}^{-\mathrm{i} k_{j, f e} L_{x}}$, the corresponding wavenumber $k_{j}$ can be given by $k_{j, f e}=\frac{\log \gamma_{j, f e}}{-\mathrm{i} L_{x}}$ which can be directly compared to the reference wavenumber values $k_{j, r f}$. The objective function of the identification process to be minimized is then obtained through a least squares approach as

$$
\mathcal{F}(\mathbf{p})=\sum_{m=1}^{m_{\max }}\left(k_{m, r f}-k_{m, f e}\right)^{2}
$$

with $k_{m, r f}$ and $k_{m, f e}$ being measured and calculated respectively at frequency $\omega_{m}$ for the same wave type, while $\mathbf{p}$ is the vector of parameters to be identified; in the very general case this is expressed as

$$
\mathbf{p}=\left\{E_{x, 1} E_{y, 1} E_{z, 1} v_{x y, 1} v_{x z, 1} v_{y z, 1} G_{x y, 1} G_{x z, 1} G_{y z, 1} h_{1} \rho_{m, 1} \cdots \rho_{m, l_{\max }}\right\}^{\top}, \quad l \in\left[1, l_{\max }\right]
$$

In the above, $m_{\max }$ is the total number of reference eigenvalues which can be used in the identification procedure. 
To accelerate the Newton-like iterative scheme, the first (or even the second) gradient of the objective function $\frac{\partial \mathcal{F}}{\partial \beta_{i}}$ may be provided for each sought structural property $\beta_{i}$ as

$$
\frac{\partial \mathcal{F}}{\partial \beta_{i}}=\sum_{m=1}^{m_{\max }}\left(2 k_{m, f e} \frac{\partial k_{m, f e}}{\partial \beta_{i}}-2 k_{m, r f} \frac{\partial k_{m, f e}}{\partial \beta_{i}}\right)
$$

It is noted that the set of parameters may be considered to have constrained values (e.g. $\left.\beta_{i} \in\left[\beta_{i, \min }, \beta_{i, \max }\right]\right)$, again for practical reasons.

The constrained minimization problem can be implemented within standard mathematics software and nonlinear optimization algorithms (such as fmincon in MATLAB) can be employed in order to compute the optimal parameter vector $\mathbf{p}$ that minimizes $\mathcal{F}(\mathbf{p})$ and corresponds to the identified structural properties.

\section{Numerical and experimental case studies}

\subsection{Numerical validation of the identification scheme}

Numerical calculations are made on a layered composite structural configuration. A Hanning window was applied at all pulses with $n_{0}=9$. The results for six wave pulses of different frequencies are presented in Fig.3.

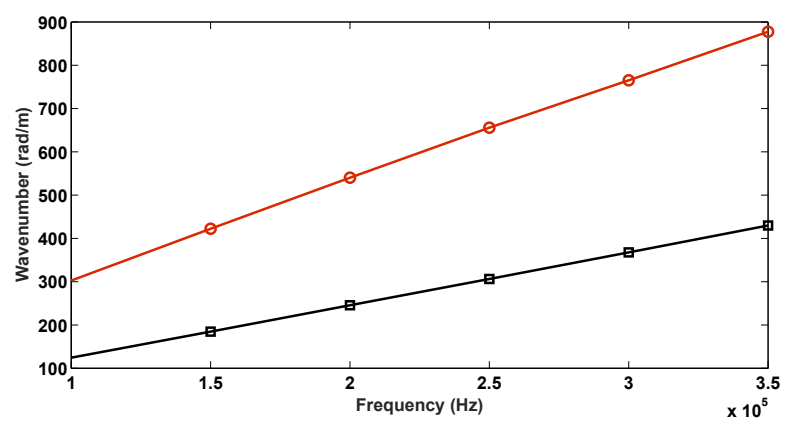

Figure 3. Reference wavenumber values obtained through a numerical solution of the full FE model for the multilayer structure. Results for a pressure propagating wave $(\square)$ and a flexural propagating one (O).

In order to find out the maximum number of parameters that can be identified within a rational amount of time for the multilayer structure, we run the identification procedure for three, four, five and six unknown parameters. It was observed that the processes with five and six unknown parameters never converged to a satisfactory value of the objective function after 18,000 s of post-processing time ( 5 hours). This is due to the existence of an important number of local minima that needed to be investigated by the fmincon algorithm. None of the derived local solutions however had a value close to zero.

The identification process did converge when four parameters were considered unknown $\left(\rho_{l s}, G_{x z, c}, E_{x, u s}\right.$ and $\left.h_{u s}\right)$ with c, ls and us the core, lower and upper skins (facesheets) indices. The minimization process converged after 137 iterations each of which lasted approximately 14 seconds, resulting in a total computation time of 1950 s on a laptop device. The properties identified through the results corresponding to the pressure wave are presented as

$$
\rho_{\mathrm{ls}}=3474.8 \mathrm{~kg} / \mathrm{m}^{3} \quad G_{\mathrm{xz}, \mathrm{c}}=35.44 \mathrm{MPa} \quad E_{\mathrm{x}, \mathrm{us}}=148.91 \mathrm{GPa} \quad h_{\mathrm{us}}=1.0038 \mathrm{~mm}
$$


Very good agreement exists between the recovered values and the ones initially injected in the full FE model (maximum divergence not greater that $1 \%$ ), while the final value of the objective function was equal to 5.34. It is evident that incorrect wavenumber measurements will radically increase the value of the calculated objective function, therefore leading to a non-convergent problem.

\subsection{Structural identification through experimental measurements for a layered composite}

The structure is a rectangular sandwich plate placed in a horizontal position as depicted in Fig.(4). The sandwich's skins are $0.6 \mathrm{~mm}$-thick Hexforce with multi-axial, carbon-reinforced fibres. The density of the skins given by the manufacturer is $\rho_{s}=1451 \mathrm{~kg} . \mathrm{m}^{-3}$ and the core's density is $\rho_{c}=99 \mathrm{~kg} \cdot \mathrm{m}^{-3}$.

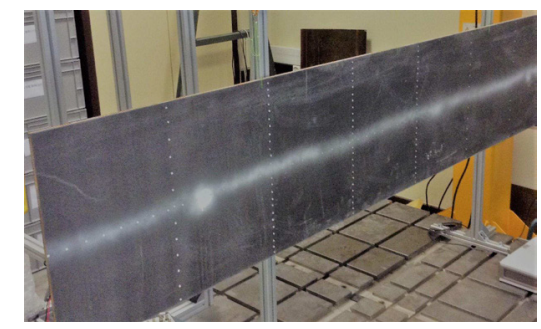

Figure 4. Caption of the structure used to retrieve experimental wavenumbers.

Static measurements conducted on the layered structure provides the following mechanical characteristics:

$$
E_{\text {manuf }}=70 \mathrm{GPa} \text { and } G_{\text {manuf }} \in[30-38] \mathrm{MPa}
$$

The shaker is used to produce the harmonic excitation at the edge of the plate while the laser vibrometer is used to measure normal displacement field. The phase velocities obtained by the IWC method are shown in Fig.5. Material properties obtained from the IWC characterization [18] are:

$$
E_{\mathrm{IWC}}=62 \mathrm{GPa} \text { and } G_{\mathrm{IWC}}=37.8 \mathrm{MPa}
$$

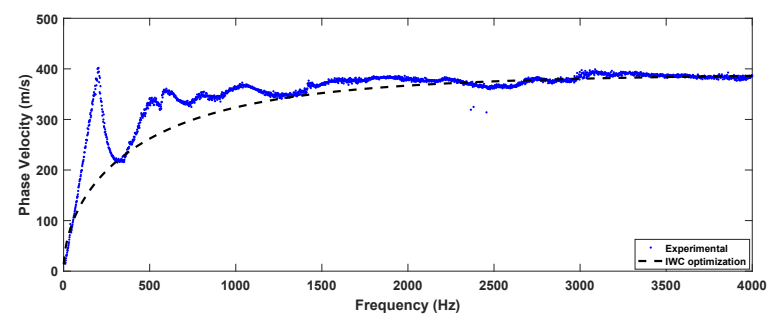

Figure 5. Flexural phase velocities obtained in the main direction of the plate. The convergence however increases with frequency.

Note that a refined frequency sampling was used in [18] to evaluate the transition frequency at $880 \mathrm{~Hz}$, and retrieve the following material characteristics:

$$
E_{\mathrm{TFC}}=69.8 \mathrm{GPa} \text { and } G_{\mathrm{TFC}}=36.5 \mathrm{MPa}
$$


Taking into account the material characteristics provided by the manufacturer of the layered panel and presented above, the WFE iterative process is formed and the properties of the panel are identified through the presented, Newton-like minimization scheme. Three elements comprise the WFE model which results in very fast model updating and eigenproblem solutions for Eq.2.

The identified Young's modulus for the skins of the laminate and the shear modulus of the honeycomb core in the direction under investigation are computed as:

$$
E_{\mathrm{WFE}}=69.5 \mathrm{GPa} \text { and } G_{\mathrm{WFE}}=37.1 \mathrm{MPa}
$$

which are both in very good agreement with the values provided by the other methods mentioned above, therefore experimentally validating the exhibited computational scheme.

\section{Conclusions}

In this work we have developed and applied a new identification technique based on FE modelling and the properties of propagating waves in multilayered structures. The principal contribution resulting from this work is a robust numerical NDE procedure for recovering effective structural parameters of complex, layered composites.

\section{References}

[1] E.N. Chatzi, A.W. Smyth, Structural Control and Health Monitoring 20, 1081 (2013)

[2] C. Devriendt, P. Guillaume, Mechanical Systems and Signal Processing 21, 2689 (2007)

[3] T. Kijewski, A. Kareem, Computer-Aided Civil and Infrastructure Engineering 18, 339 (2003)

[4] A. Karakoç, J. Freund, Composite Structures 94, 2017 (2012)

[5] M. Matter, T. Gmür, J. Cugnoni, A. Schorderet, Composite Structures 93, 331 (2011)

[6] D.J. Mead, Journal of Sound and Vibration 27, 235 (1973)

[7] B.R. Mace, D. Duhamel, M.J. Brennan, L. Hinke, The Journal of the Acoustical Society of America 117, 2835 (2005)

[8] J.M. Mencik, M. Ichchou, European Journal of Mechanics-A/Solids 24, 877 (2005)

[9] D. Chronopoulos, M. Ichchou, B. Troclet, O. Bareille, Composite Structures 107, 149 (2014)

[10] D. Chronopoulos, M. Ichchou, B. Troclet, O. Bareille, Applied Acoustics 74, 1394 (2013)

[11] D. Chronopoulos, Journal of Sound and Vibration 355, 322 (2015)

[12] D. Chronopoulos, I. Antoniadis, T. Ampatzidis, Extreme Mechanics Letters 12, 48 (2017)

[13] D. Chronopoulos, Composite Structures (2017)

[14] D. Chronopoulos, M. Ichchou, B. Troclet, O. Bareille, Aerospace Science and Technology 30, 192 (2013)

[15] R. Apalowo, D. Chronopoulos, M. Ichchou, Y. Essa, F. Martin De La Escalera, Proceedings of the Institution of Mechanical Engineers, Part C: Journal of Mechanical Engineering Science 231, 3042 (2017)

[16] D. Chronopoulos, D. Chronopoulos, M. Collet, M. Collet, M. Ichchou, M. Ichchou, Engineering Computations 34, 1572 (2017)

[17] D. Chronopoulos, C. Droz, R. Apalowo, M. Ichchou, W. Yan, Composite Structures 182, 566 (2017)

[18] C. Droz, O. Bareille, M.N. Ichchou, Composites Part B: Engineering 112, 103 (2017) 